\title{
La gouvernance de l'eau souterraine dans le Sahara algérien : enjeux, cadre légal et pratiques locales
}

\author{
Ali Daoudi ${ }^{1,2}$, Caroline Lejars ${ }^{3,}$ et Nesrine Benouniche ${ }^{1}$ \\ ${ }^{1}$ École nationale supérieure d'agronomie d'Alger (ENSA), avenue Hassan Badi, El Harrach, Alger, Algérie \\ ${ }^{2}$ Centre de recherche scientifique et technique sur les régions arides (CRSTRA), université Mohamed Khider, Biskra, Algérie \\ ${ }^{3}$ CIRAD, UMR G-EAU, 34398 Montpellier, France
}

\begin{abstract}
Résumé - Depuis la découverte de son grand potentiel hydrique, et la baisse du coût de mobilisation de l'eau souterraine, le Sahara, où existait une agriculture oasienne en difficulté, est considéré comme l'espoir de l'agriculture algérienne. Sous l'effet conjugué de politiques publiques généreuses, notamment en matière de réalisation d'infrastructures, et de dynamiques locales novatrices, une nouvelle agriculture saharienne, intensive et forte consommatrice d'eau, s'est développée. C'est le cas de la wilaya de Biskra en Algérie où, depuis une vingtaine d'années, des palmiers en monoculture et des serres tunnels s'étendent à perte de vue en marge des oasis traditionnelles. Dans cet article, nous analysons un des déterminants clés de ce développement : le système de gouvernance de l'eau. Nous nous focalisons sur le rôle de l'État, des acteurs privés et leurs interrelations dans l'exploitation de la ressource hydrique et nous analysons les configurations institutionnelles et réglementaires qui affectent le rapport des agriculteurs à la ressource en eau. Nous montrons (i) et que les pratiques locales incitent à valoriser l'eau à la parcelle et à maximiser les prélèvements à l'échelle d'un forage (ii) que même s'il existe un décalage entre le cadre réglementaire et les pratiques locales, l'État est « tolérant » mais non absent et pourrait reprendre la main pour réguler l'usage à l'échelle régionale.
\end{abstract}

Mots clés : irrigation / gouvernance / Sahara / eau souterraine / Algérie

\begin{abstract}
Groundwater governance in Algerian Sahara: challenges, legal framework and local practices. Since the discovery of its large water potential, the Sahara, where the oasis agriculture was in difficulty, has been considered as an opportunity for Algerian agriculture. In some regions, the combined effects of ambitious public policies and local dynamics have deeply changed the landscape of the Saharan oases. In Algeria, this is the case of the municipality of El Ghrous in the region of Biskra, where for 20 years, palm tree monoculture and tunnel greenhouses have dramatically expanded on the margins of traditional oases. In this article, we analyze one of the key factors that promote and threaten this agricultural expansion: the groundwater governance system. We focused on the role of the State and private actors, and their relationships. We analyzed more specifically water governance focusing on the interaction between the resource, farmers, public and private suppliers of water infrastructures and institutions regulating access to water and land. We show that the adaptability of this agriculture is mainly the result of a complex set of actors, at bilateral and collective levels, who manage to reconfigure and adapt the institutional framework of water governance.
\end{abstract}

Keywords: irrigation / governance / Sahara / groundwater / Algeria

\section{Introduction}

Au Sahara, l'agriculture se limite traditionnellement aux oasis, lieux de production et d'habitation, dont l'existence est liée à des sources d'eau relativement accessibles (oueds, sources artésiennes, foggaras). L'eau, composante constitutive

\footnotetext{
$\bar{*}$ Auteur de correspondance : caroline.lejars@cirad.fr
}

de ces oasis, y est gérée collectivement grâce à une matrice institutionnelle endogène (droits de propriété, règles de transmission et d'accès, instruments et méthodes de mesure, etc.) qui a fait ses preuves des siècles durant, en matière de coordination des interactions interindividuelles autour de l'accès à cette ressource (Marouf, 1980 ; Granier, 1980).

À partir des années 1980, la technique de forage s'est généralisée et son coût a considérablement baissé, conduisant ainsi à une multiplication phénoménale des forages individuels, 
base de développement d'une nouvelle agriculture saharienne, en dehors des oasis, intensive et totalement orientée vers le marché (Mubarak, 1998 ; Côte, 2002). Cette multiplication des forages individuels soulève toutefois des inquiétudes quant au risque de surexploitation des nappes souterraines et recentre le débat de la mise en valeur agricole dans les régions arides autour de la question de la gouvernance de l'eau, comme condition nécessaire à la durabilité de ces activités (Kuper et al., 2016; Petit et al., 2017).

Le risque lié à la surexploitation des eaux souterraines (au sens de prélèvement supérieur à la recharge) nécessite de mettre en place une gouvernance qui garantit une allocation efficace, équitable et durable de la ressource (Petit, 2004 ; Llamas et Martinez-Santos, 2012 ; Jenifer et Arul, 2012). Deux grandes configurations sont souvent distinguées, en fonction de leur degré de centralisation des mécanismes d'allocation de l'eau. La première fait la part belle au marché, considéré comme étant le meilleur mécanisme pour une affectation efficace de l'eau, à travers un prix s'imposant à tous et qui reflète le niveau de rareté de la ressource (Petit, 2004). Cette option exige l'existence, au préalable, de droits de propriété privée de l'eau socialement acceptés. La seconde option met l'État, considéré comme le meilleur garant de l'intérêt général, au centre du dispositif d'allocation de l'eau. L'État, propriétaire de la ressource, régule son allocation à travers des mécanismes réglementaires (autorisation, permis) et économiques (redevances) (Petit, 2004). Entre ces deux grandes configurations, l'analyse de la gouvernance des eaux souterraines a donné naissance à plusieurs approches théoriques et méthodologiques, que Faysse et Petit (2012) regroupent en trois catégories principales. La première approche est celle de la gestion collective de la ressource développée par Ostrom $(1990,1992)$ qui met en évidence la capacité des collectifs à s'auto-organiser pour construire des règles de gestion et conduire une action collective permettant, sous certaines conditions, une gestion durable des ressources communes. La deuxième est celle proposée par Shah (2009), en Asie du Sud, dans un contexte «d'anarchie » face à l'abandon ou à l'incapacité de la puissance publique de proposer une forme de planification des règles d'accès et des usages qui serait soutenable, en particulier au plan environnemental. Dans ce contexte, l'absence de l'État favoriserait l'émergence d'arrangements institutionnels autour desquels les acteurs privés structurent eux-mêmes leurs règles définissant l'accès à l'eau. À l'opposé des conclusions d'Ostrom, Shah considère que ces arrangements institutionnels ne contribuent pas à une gestion durable de la ressource, et propose de limiter les prélèvements par des leviers indirects, comme l'augmentation du prix de l'énergie. La troisième approche est celle de la political ecology, qui met davantage l'accent sur les enjeux et leviers sociopolitiques liés à l'accès et l'usage de l'eau souterraine, et ce en analysant les rapports de force entre les différents acteurs impliqués dans la gouvernance de l'eau. L'enjeu de la surexploitation n'est pas central dans les travaux des auteurs de cette approche, cités par Faysse et Petit (2012).

En Algérie, la gouvernance de l'eau associée au développement agricole se rapproche singulièrement de celle décrite par Shah (2009) en Asie. Le Sahara algérien a connu, ces 30 dernières années, une extension rapide de la nouvelle agriculture saharienne, résultat d'un processus de déverrouil- lage de l'accès aux facteurs de production (foncier et à l'eau), fortement soutenu par l'État (Otmane et Kouzmine, 2013 ; Daoudi et al., 2015 ; Hamamouche et al., 2015 ; Daoudi et Lejars, 2016). Entre 1983 et 2015, plus de 1,4 millions d'hectares de terres non cultivées ont été affectés par l'État à des dizaines de milliers de candidats à la mise en valeur, au sens de « mise en culture irriguée » (Bessaoud, 2013 ; Daoudi et al., 2015). L'Algérie est ainsi classée parmi les principales régions d'utilisation intensive des eaux souterraines pour l'agriculture (Siebert et al., 2010). D'un point de vue réglementaire (loi sur l'eau de 2005), l'accès à l'eau souterraine sur les terres mises en valeur est soumis à autorisation. Toutefois, l'application de la loi semble limitée et les forages illicites (sans autorisation) sont plus nombreux que les forages autorisés. Comme dans d'autres pays du Maghreb, pour les agriculteurs, si l'eau souterraine reste un « bien de l'État» (dont l'accès est soumis à autorisation), c'est aussi un « bien et un don de Dieu » pour « une eau à soi » (Kuper et al., 2012 ; Quarouch et al., 2014). L'absence de cadre institutionnel consensuel admis et partagé par tous les usagers locaux, le décalage entre la politique agricole (qui encourage la mise en valeur) et la politique de l'eau, ainsi que l'écart entre le cadre formel et les pratiques locales en matière de gestion de l'eau, mettent en évidence l'incapacité de la puissance publique à proposer des règles d'accès et d'usage qui soient soutenables et partagées. Dans ce cadre, les acteurs privés ont structuré eux-mêmes leurs règles d'accès à l'eau et au foncier autour de nombreux arrangements informels (Amichi et al., 2015).

Dans cet article, nous présentons les résultats d'une étude menée dans la commune d'El Ghrous (wilaya de Biskra), portant sur l'analyse des systèmes de gouvernance de l'eau souterraine en rapport avec l'extension rapide de la mise en valeur agricole. Nous proposons une analyse du cadre réglementaire formel régissant l'accès et l'usage de l'eau et une caractérisation des pratiques locales autour de l'eau d'irrigation dans les nouveaux périmètres agricoles de la commune. Nous montrons :

- que les pratiques locales incitent à économiser l'eau à la parcelle et à maximiser les prélèvements à l'échelle d'un forage ;

- que même s'il existe un décalage entre le cadre réglementaire et les pratiques locales, l'État est « tolérant » (pour reprendre l'expression utilisée par Brochier-Puig [2004]) mais non absent, et pourrait reprendre la main pour réguler l'usage à l'échelle régionale.

\section{Contexte, zone d'étude et méthodologie}

\subsection{Site de l'étude : Biskra et la commune d'EI Ghrous}

Biskra est l'une des wilayas sahariennes où la mise en valeur des terres a permis un développement agricole spectaculaire sur un large front pionnier (Petit et al., 2017). La néo-agriculture saharienne (par opposition à l'agriculture oasienne traditionnelle) a connu un développement fulgurant, de type front pionnier, particulièrement grâce aux cultures maraîchères sous serre plastique (plasticulture) qui se sont développées dans certaines parties de la wilaya, en parallèle à la phœniciculture, sur de nouvelles terres mises en culture 


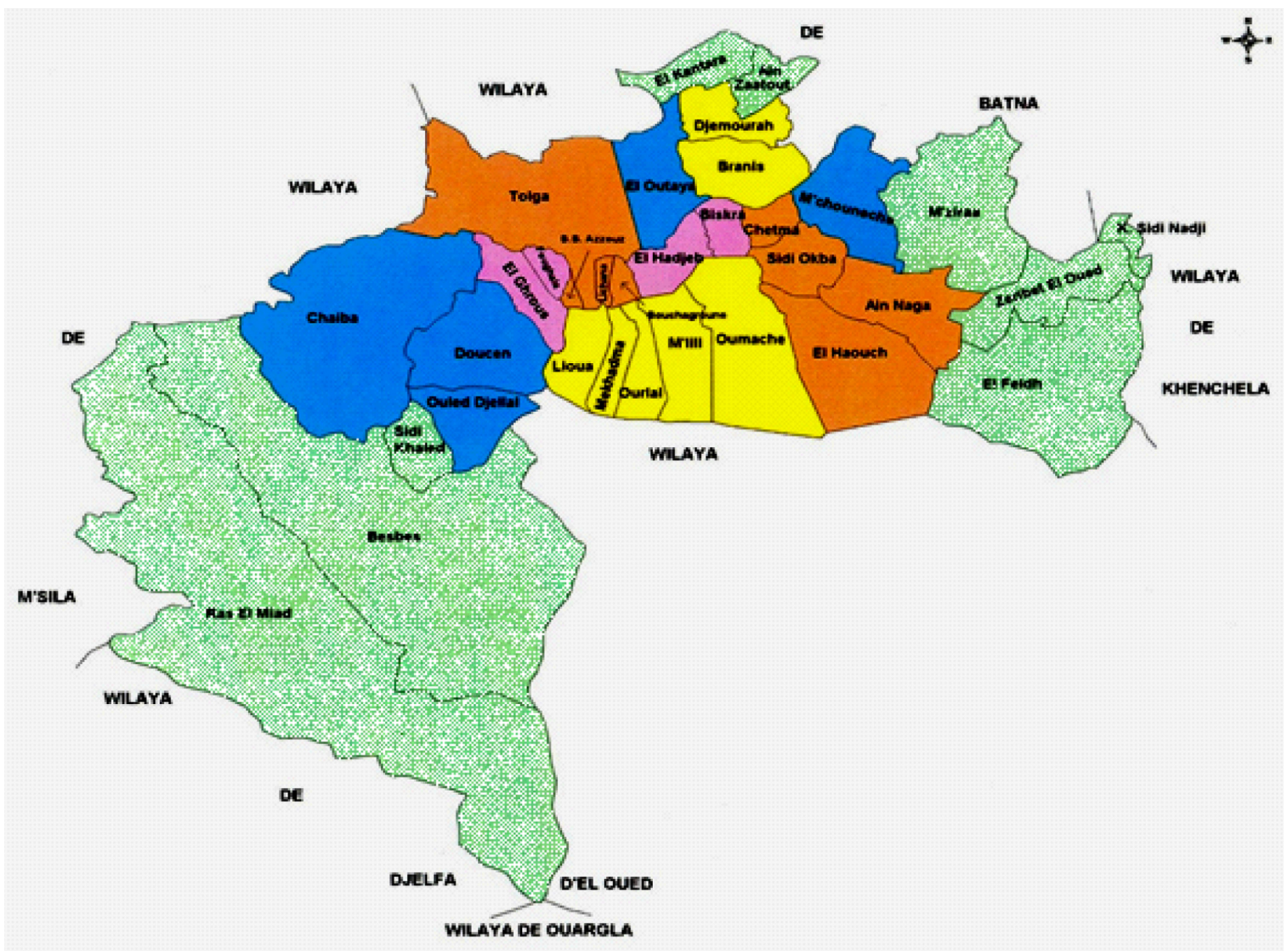

Fig. 1. Localisation de la zone d'étude. Source : Direction des services agricoles de Biskra.

Fig. 1. Location of study area.

(Khiari, 2002 ; Bouammar, 2010). La wilaya dispose de plus de 100000 ha irrigués (DSA, 2013) mais le nombre exact de forages exploités est inconnu. L'antenne locale de l'agence des bassins hydrographiques du Sud estime le nombre total des forages de la wilaya à 17000 , dont 8000 seulement réalisés avec l'autorisation des autorités compétentes. Selon cette même source, la quantité d'eau pompée annuellement est estimée à 1,8 milliard de mètres cubes alors que le volume optimal exploitable (sans menacer la durabilité de la ressource) serait de 800 millions de mètres cubes par an.

L'étude a été réalisée dans la commune d'El Ghrous, située à l'Est de la wilaya de Biskra (Fig. 1), où la superficie occupée par la plasticulture a augmenté de $79 \%$ entre 2001 et 2012, pour atteindre 1049 ha de serres (APC d'El Ghrous, 2012), soit un tiers des serres de la wilaya. La phœniciculture a également connu un développement important durant la période considérée, sa superficie est passée de 1285 ha à 1955 ha (DSA, 2013). Cette extension s'est faite :

- à l'initiative des autorités locales (commune ou wilaya), sur des terres publiques aménagées par l'État (délimitation et partage en lots, installation de forages collectifs, ouverture de pistes, électrification) et attribuées à des particuliers dans le cadre des programmes de concession ou de la loi APFA encadrant l'accès à la propriété foncière par la mise en valeur agricole ;

- à l'initiative des particuliers sur des terres publiques, appropriées privativement, dont la mise en valeur a été totalement prise sur fonds propres (la mise en valeur sur initiative des particuliers est toutefois généralement régularisée dans le cadre de la loi de l'APFA ou des concessions [Bouammar, 2010 ; Daoudi et al., 2015]).

La dynamique d'extension a également été stimulée par la maîtrise de la technique de forage par des entrepreneurs privés locaux et la réduction de son coût (Bensaâd, 2011). Le dernier bilan (2013) de la direction des ressources en eau de la wilaya fait état de 470 puits et 511 forages sur la commune, alors que les acteurs locaux (agriculteurs et autorités locales) estiment le nombre des forages à plus de 1000 , la moitié étant illicites (Daoudi et Lejars, 2016). 


\subsection{Cadre d'analyse de la gouvernance de l'eau et enquêtes réalisées}

Dans ce travail, nous considérons la gouvernance de l'eau comme l'ensemble des formes de coordination entre acteurs en matière d'allocation et de gestion de l'eau (Faysse et Petit, 2012). Ces formes de coordination concernent des actions individuelles, réalisées dans un cadre marchand ou non, des actions collectives, engagées par des groupes d'individus partageant des intérêts communs, et des actions des pouvoirs publics (Petit, 2004).

L'analyse vise à mettre en évidence les décalages et les interactions entre les pratiques locales et le cadre légal. Dans cet article, nous n'entrons pas dans la description détaillée des arrangements, au sens de la nature et structure du rapport contractuel qui relie les acteurs entre eux (rapport salarial, rapport au foncier, rapport à l'eau), ceux-ci ayant été décrits par Amichi et al. (2015) dans le cas de forages privés. Nous nous focalisons ici sur l'interaction entre les pratiques formelles et les pratiques locales, y compris sur les forages collectifs dans les périmètres de mise en valeur.

L'analyse des actions de l'État est focalisée sur les droits de propriété et d'usage définis dans la loi sur l'eau de 2005 $\left(\mathrm{n}^{\mathrm{o}}\right.$ 05-12 de 2005 relative à l'eau). Pour comprendre l'évolution et l'adaptation de ces droits, notamment par rapport aux changements enregistrés sur le terrain, le contenu de la loi de 2005 est comparé à celui du premier Code de l'eau en Algérie, la loi 83-17 de 1983.

La caractérisation des pratiques locales est centrée :

- sur l'analyse des arrangements pour la réalisation des forages, des usages effectifs de l'eau par les agriculteurs, et des pratiques d'échanges marchands ;

- sur une comparaison des pratiques locales avec le cadre réglementaire.

Cette caractérisation s'est appuyée sur les résultats d'entretiens réalisés, entre 2014 et 2015 , avec 100 propriétaires de forages (individuels ou collectifs), dix agriculteurs locataires, deux chefs d'entreprise de forage, des agents de l'antenne de Biskra de l'Agence des bassins hydrographiques du Sahara. L'enquête auprès des propriétaires et usagers de forages s'est faite dans des zones à vocation phœnicicole et dans des zones de mise en valeur où domine la plasticulture (périmètres de concession, d'APFA et de mises en valeur privée régularisée dans le cadre de l'APFA ou la concession).

\section{Résultats}

\subsection{Droits de propriété et d'usage : ce que prévoit la loi sur l'eau}

L'eau souterraine fait partie du domaine public hydraulique et est donc propriété publique de l'État (Code de l'eau de 1983). Son usage a été codifié pour la première fois depuis l'Indépendance en 1983 à travers la loi 83-17. Cette loi stipule que tout usage de l'eau souterraine est soumis à autorisation des autorités compétentes.

Toutefois, cette loi ne fait pas explicitement référence à l'utilisation de l'eau souterraine pour l'irrigation, la partie irrigation de la loi se focalisant sur l'eau des barrages. La faible référence aux eaux souterraines dans cette première loi sur l'eau contraste avec les orientations de la loi 83-18 portant sur l'accès à la propriété foncière par la mise en valeur agricole (APFA), adoptée la même année. En effet, la loi de l'APFA encourage la mise en valeur de terres autrefois consacrées à un usage agricole extensif (terre de parcours steppiques) ou non utilisées pour l'agriculture (terres sahariennes). Cette « mise en valeur » est entendue au sens de " mise en culture irriguée », nécessairement par les eaux souterraines au Sahara. Le contenu de ces deux lois montre le décalage entre les politiques agricole et hydraulique.

Le code de l'eau de 1983, principalement focalisé sur les eaux superficielles, s'inscrit dans la doctrine d'utilisation planifiée de l'eau, une planification censée promouvoir une utilisation rationnelle de la ressource en se basant sur deux instruments : le cadastre hydraulique (inventaire des données de base relatives aux ressources en eau) et l'équilibre hydrique (confrontation entre les ressources en eau et les différents besoins). Le territoire national est découpé en unités hydrographiques naturelles dénommées bassins hydrographiques, au niveau desquels la conservation qualitative et quantitative des ressources en eau est conçue (articles 130 et 131). Le paiement de redevance d'exploitation n'est pas mentionné dans cette loi ; le principe de gratuité de l'accès à l'eau serait donc implicite.

En 2005, une nouvelle loi sur l'eau a été adoptée, réservant une place importante à la réglementation de l'utilisation de l'eau souterraine, notamment pour l'irrigation. Si cette loi confirme le caractère prioritaire de l'agriculture (après les besoins en eau potable), elle marque une rupture avec la gestion planifiée et étatique de la ressource. Elle introduit de nouveaux principes sur lesquels se fondent l'utilisation et la gestion durable des ressources en eau, notamment la prise en compte des coûts réels des services d'approvisionnement en eau à usage domestique, agricole et industriel et la systématisation des pratiques d'économie et de valorisation de l'eau et de lutte contre les pertes et le gaspillage (article 3).

La loi de 2005 reconnaît aux détenteurs de droits sur une terre agricole un droit d'usage de l'eau souterraine, dont l'accès par forage est soumis à autorisation, pour les nappes renouvelables, ou à concession pour les nappes non renouvelables. La réalisation d'un forage sans autorisation ou concession est punie d'emprisonnement et d'amendes.

L'octroi d'une autorisation ou d'une concession par le Wali (gouverneur), sur avis des services techniques compétents, pour réaliser un forage est conditionné :

- par l'existence de besoins à satisfaire justifiés ;

- par l'absence de préjudice à la protection quantitative des nappes ;

- par l'absence de préjudice à l'intérêt général ;

- par l'absence de préjudice aux droits des tiers dûment établis.

Ces conditions remplies, le demandeur obtient un droit d'usage qui lui confère " la disposition, pour une durée déterminée, d'un débit ou d'un volume d'eau déterminé sur la base des ressources globales disponibles en année moyenne et des besoins correspondant à l'usage considéré » (article 72).

La loi exige ensuite du titulaire du droit d'usage (article 87):

- d'utiliser l'eau d'une façon rationnelle et économique ;

- d'installer des dispositifs de mesure et de comptage des consommations d'eau ; 


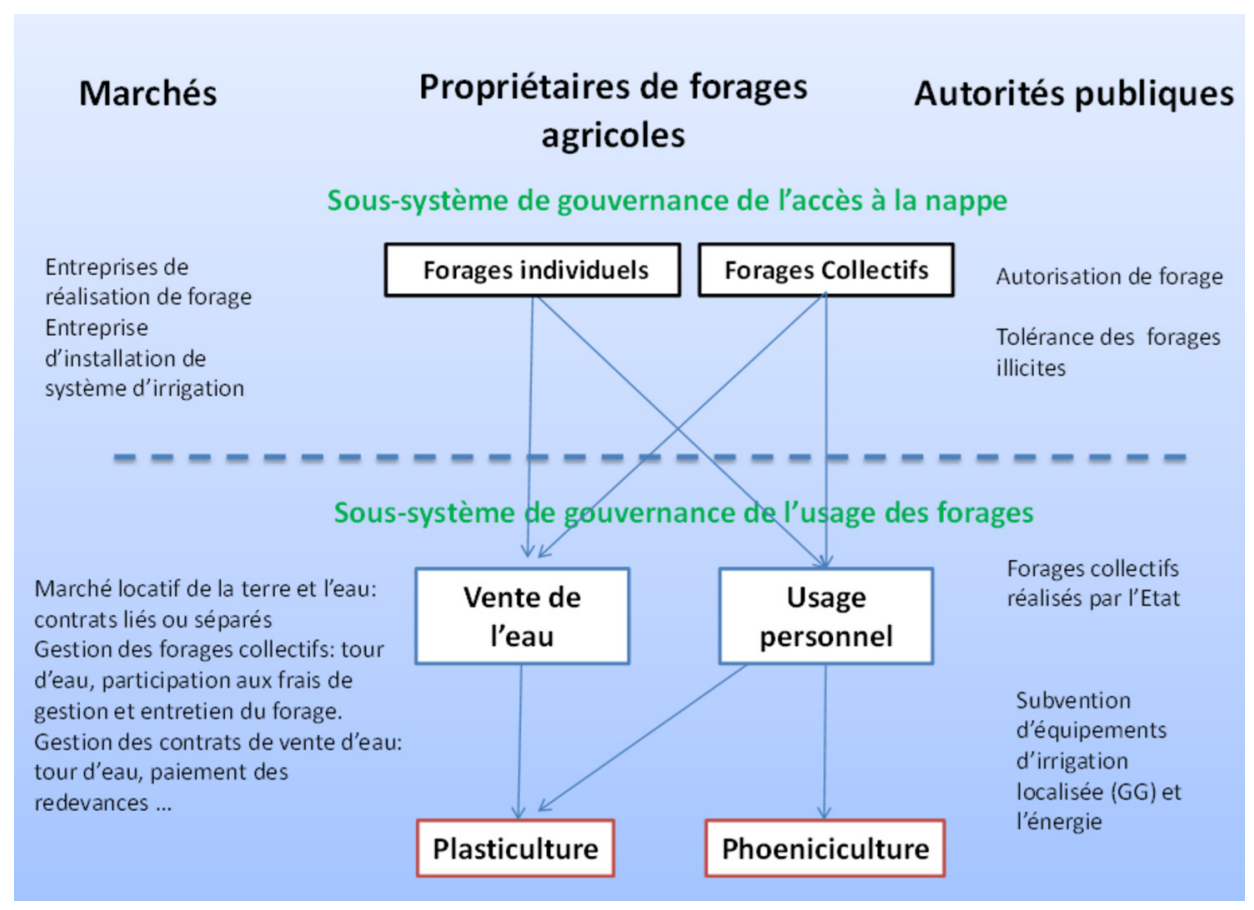

Fig. 2. Schéma de gouvernance des forages dans la commune d'El Ghrous.

Fig. 2. Governance of drillings and wells in the municipality of El Ghrous.

- de se soumettre aux opérations de contrôle effectuées par les agents habilités.

Elle oblige explicitement les usagers de l'eau d'irrigation à utiliser des techniques permettant d'économiser l'eau et prévoit la suspension, voire l'annulation, du droit d'usage en cas de constatation de gaspillage.

Enfin, la loi de 2005 introduit l'obligation du paiement d'une redevance en contrepartie du droit d'usage de l'eau souterraine. La valeur de la redevance, qui devait être définie par voie réglementaire, n'est toujours pas appliquée au secteur agricole.

La loi de 2005 semble donc s'inscrire dans la doctrine de gestion intégrée des ressources en eau, telle que définie à l'échelle internationale par des organismes comme le Partenariat mondial de l'eau (Petit, 2016). Cette gestion cible un compromis théorique entre l'exploitation des ressources hydriques disponibles et leur préservation, compromis conceptualisé à travers le concept d'exploitation rationnelle et durable. L'opérationnalisation de ces deux concepts nécessite une bonne maîtrise des différents aquifères exploités (limites, stocks, recharge) ainsi qu'un contrôle précis et rigoureux de leur exploitation (nombre de forages, périodes de pompage, volumes prélevés).

Les observations de terrain montrent que les autorités chargées de la gestion des nappes souterraines sont loin d'avoir une maîtrise satisfaisante des outils de gestion de ces nappes (cadastre et balance hydrauliques), et l'application des dispositions de la loi de 2005 reste très limitée. Cet écart n'est pas propre à l'Algérie, il est constaté dans la plupart des pays qui proposent les mêmes instruments (Petit, 2004).

\subsection{Accès et utilisation de l'eau d'irrigation : les pratiques locales}

Sur le terrain, les pratiques des acteurs sont différentes et complexes, obéissant à la fois à des règles du jeu formelles et informelles.

À El Ghrous, et à Biskra d'une manière générale, le système de gouvernance de l'eau souterraine peut être scindé en deux sous-systèmes aux logiques et règles de fonctionnement différentes (Fig. 2). Le premier sous-système concerne l'accès aux nappes souterraines, exploitées à travers des forages le plus souvent réalisés par les agriculteurs euxmêmes, et le second est relatif à la gestion des forages et à l'usage de l'eau à la parcelle.

Dans le premier sous-système, l'État est un acteur central. Selon les personnes ressources rencontrées, les décalages observés entre le cadre légal et les pratiques locales (Tableau 1) sont, en grande partie, tolérés intentionnellement par les pouvoirs publics. Ces derniers durcissent ou relâchent la délivrance des autorisations de forage ou le contrôle des forages illicites, en fonction des besoins en développement agricole local, de la situation politique et de l'état de la ressource.

Les entretiens ont en effet montré qu'à l'époque de la mise en œuvre du plan national de développement agricole et rural (2000-2006), considéré comme une priorité nationale, les forages autorisés ou illicites se sont considérablement développés. Après cette période, l'octroi des autorisations s'est durci et la tolérance aux forages illicites dépend aujourd'hui, d'une zone à une autre, de l'état de la ressource et, d'une période à l'autre, des tensions sociales et politiques au niveau local. 
Tableau 1. Écarts entre les dispositions du cadre légal (loi sur l'eau de 2005) et les pratiques locales d'accès et d'usage de l'eau. Table 1. Differences between legal framework (Water Act 2005) and local practices for accessing and using water.

\begin{tabular}{lll}
\hline & Dispositions de la Loi sur l'eau de 2005 & Pratiques locales observées \\
\hline Propriétaire & Domaine public hydraulique & $\begin{array}{l}\text { Perception ambiguë. « l'eau, c'est moi qui la } \\
\text { mobilise, j'engage beaucoup d'argent pour le } \\
\text { faire, elle m'appartient » }\end{array}$
\end{tabular}

Droit d'usage

Formes de cession des droits d'usage

Enforcement

Paiement des droits d'usage

Restrictions aux droits d'usage
« .. confère à son titulaire la disposition, pour une durée déterminée, d'un débit ou d'un volume d'eau déterminé sur la base des ressources globales disponibles en année moyenne et des besoins correspondant à l'usage considéré » (article 72)

Autorisation (nappe renouvelable) concession (nappe fossile ou faiblement renouvelable)

- des besoins à satisfaire justifiés

- la satisfaction de ces besoins ne porte pas préjudice à la protection quantitative et qualitative des ressources en eau

- la réalisation du forage ne lèse pas l'intérêt général - la réalisation du forage ne porte pas préjudice aux droits des tiers dûment établis

L'autorisation ou la concession est accordée au profit d'un fonds déterminé. En cas de cession du fonds, le droit d'utilisation est transféré de plein droit au nouveau propriétaire (article 128)

Toute réalisation de forage sans autorisation ou concession est punie d'un emprisonnement et d'une amende. Les équipements et matériels ayant servi à commettre l'infraction peuvent être confisqués

L'autorisation et la concession donne lieu au paiement d'une redevance

Les usagers de l'eau n'ont le droit de l'exploiter que dans la limite de l'objet pour lequel elle est destinée (article 15).

L'autorisation et la concession sont révocables (article 87) si son titulaire ne respecte pas l'obligation (article 16) :

- d'utiliser l'eau d'une façon rationnelle et économique

- d'installer les dispositifs de mesure et de comptage des consommations d'eau

- de se soumettre aux opérations de contrôle effectuées par les agents habilités

Les irrigants sont tenus d'assurer une valorisation optimale des ressources en eau (article 60)
Aucune limitation de durée ou de volume ou de type d'usage, y compris pour la vente

Autorisation pour forage individuel ou collectif

Accès informel à la nappe (réalisation de forage sans autorisation)

Exigence d'une superficie minimale (10 ha) pou obtenir une autorisation, sachant que dans la région une exploitation moyenne varie entre 2 et 5 ha. Les autorités locales encouragent les agriculteurs à réaliser des forages collectifs Le délai d'étude des dossiers est lent (plusieurs mois, voire plusieurs années)

Aucun cas d'application de cette mesure n'a été mentionné par les personnes interrogées Les prix de réalisation des forages varient en fonction de la présence ou non d'autorisation. Les foreurs justifient cette augmentation par le risque de confiscation du matériel et les coûts de l'indulgence des autorités locales compétentes (corruption)

Non appliquée

Les propriétaires de forage utilisent l'eau pour l'irrigation et peuvent la vendre à d'autres agriculteurs Aucun cas de révocation n'a été relevé par les personnes interrogées

Le contrôle de l'utilisation des forages est totalement absent

Pour les agriculteurs, le forage et son eau sont des biens privés dont la gestion relève de leur seule prérogative
Dans ce premier sous-système, certains acteurs privés, principalement les entrepreneurs de réalisation de forages, peuvent jouer un rôle dans l'aboutissement des demandes d'autorisation, mais aussi dans la réalisation des forages illicites de par leurs réseaux de connaissances auprès des autorités locales. Ils sont les intermédiaires d'un système parallèle et informel d'allocation de droit d'accès à la nappe. Ils peuvent mobiliser leurs connaissances personnelles pour faciliter les procédures légales et faire aboutir les demandes dans des délais courts par rapport à la moyenne. Dans d'autres 
cas, les mécanismes décrits par l'un des foreurs interrogés se rapprochent de pratiques de corruption. Toutefois, les agriculteurs ne parlent pas de « corruption » mais plutôt de tolérance des autorités locales (communales), conscientes de la lourdeur des procédures formelles et de l'intérêt du développement de l'irrigation pour l'économie locale. Les autorités locales toléreraient ainsi les forages illicites qui ne sont pas contestés par les voisins au moment de leur réalisation.

Le second sous-système (Fig. 2) concerne l'utilisation des forages. L'utilisation des forages, réalisés avec ou sans autorisation, obéit à une logique strictement privée qui échappe complètement au contrôle de l'État, même si ce dernier continue d'y jouer un rôle indirect à travers la subvention de l'énergie et des équipements d'irrigation (goutte-à-goutte). Cette gestion privée est aussi valable pour les forages collectifs (42 des 100 forages concernés par l'étude) qu'ils soient réalisés dans le cadre des périmètres aménagés par l'État ou par les collectifs d'agriculteurs autoorganisés en associations d'irrigants. Dans les exploitations exclusivement phœnicicoles, les forages sont généralement exploités par leurs propriétaires, individuellement ou collectivement, pour l'irrigation des palmiers dattiers. C'est dans les zones où la plasticulture est importante qu'un véritable marché privé de l'eau (au sens de Easter et al., 1999) a émergé dans le sillage du développement du marché foncier locatif (Fig. 3).

Les propriétaires des forages, dans ces zones à dominance de plasticulture, ont souvent le choix entre utiliser l'eau des forages pour irriguer leurs propres systèmes de culture, la vendre à d'autres exploitants locataires ou la partager entre ces deux options. À El Ghrous, ces trois configurations coexistent. Parmi les 58 forages individuels étudiés, $36(62 \%)$ sont exclusivement exploités par leur propriétaire et $22(38 \%)$ sont exploités par plusieurs usagers. Le forage est le centre névralgique de plusieurs exploitations agricoles, généralement tenues par des locataires (Tab. 2 ; Amichi et al., 2015). La répartition de l'eau du forage entre les exploitations qui en dépendent se fait selon un tour d'eau préalablement défini comme une composante centrale du contrat de location (Daoudi et al., 2017). Le propriétaire du forage est responsable de l'installation de l'infrastructure d'irrigation (réseau de canaux enfouis) amenant l'eau du forage jusqu'aux serres. Le système d'irrigation à l'intérieur de la serre relève de la responsabilité de l'individu exploitant la serre. Toute terre est cédée avec un tour d'eau. Les " surplus » d'eau peuvent être vendus à des voisins ou à des locataires installés sur les terres d'un voisin.

En négociant le tour d'eau, le locataire connaît d'une manière précise la durée et la fréquence des irrigations par serre auxquelles il aura droit durant la période de location. Pour ces locataires, le volume d'eau disponible pour l'irrigation est une donnée exogène fixe, qui va déterminer le choix du système de culture et des techniques d'irrigation les plus à même de valoriser au mieux la quantité disponible. Pour les propriétaires, réduire le tour d'eau par serre (fréquence et durée d'irrigation) leur permet de maximiser le nombre de serres à irriguer, et donc le potentiel d'eau à vendre. De plus, les propriétaires des forages exigent le plus souvent des locataires d'installer le goutte-à-goutte pour l'irrigation à l'intérieur des serres (Ouendeno et al., 2015). La technique du goutte-àgoutte, intégrée comme clause du contrat, est donc aujourd'hui utilisée par tous les locataires.

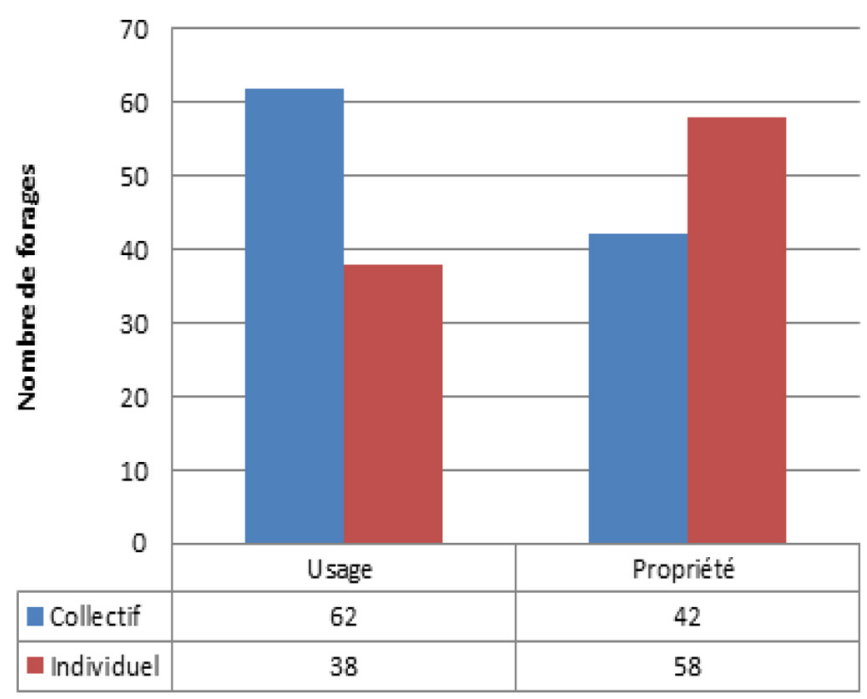

Fig. 3. Usage et propriétés des forages étudiés dans la commune d'El Ghrous. Source : nos enquêtes, 2015.

Fig. 3. Use and ownership of drillings studied in the municipality of El Ghrous.

\section{Discussion}

\subsection{Interactions entre cadre réglementaire et pratiques locales}

Dans le contexte étudié, comme dans d'autres pays du Maghreb, l'État est loin d'être absent de la régulation de l'accès et de l'utilisation de l'eau souterraine (Fofack et al., 2015). Dans une économie agricole fondée sur l'exploitation de l'eau souterraine, où l'initiative et l'investissement sont d'ordre privé, l'État participe aussi bien à la construction qu'à la mise en œuvre des règles qui organisent cette régulation. La règle officielle sur l'autorisation de forage reste en vigueur, sans être complètement restrictive. L'autorisation de forage, comme la tolérance des forages illicites, sont deux mécanismes de gestion de l'accès aux ressources hydriques souterraines parallèlement utilisés par les autorités locales compétentes, au gré des rapports de forces du moment. L'État reste ainsi présent et « régule sa tolérance » aux forages illicites.

L'application partielle du cadre réglementaire formel et son incomplétude laissent aux acteurs locaux une marge pour une coordination par des règles du jeu informelles, notamment autour des forages illicites (comme le montre également Shah [2009] en Asie). Ces règles et pratiques locales permettent de maximiser l'utilisation de l'eau à l'échelle de l'exploitation Toutefois, la non-transparence de certaines règles réduit leur légitimité et les agriculteurs locaux, notamment les petits exploitants, pour qui les coûts de transaction des forages illicites seraient prohibitifs, considèrent ces règles informelles comme peu équitables.

Enfin, les acteurs locaux gardent également la capacité de mobiliser les règles légales si leurs activités sont menacées. C'est le cas dans d'autres régions de l'Algérie (e.g. commune de Rechaiga, wilaya de Tiaret) où le niveau de rabattement de la nappe a atteint un seuil menaçant la pérennité de l'irrigation dans les exploitations existantes. Sur ces zones, l'octroi 
Tableau 2. Diversité des pratiques autour des forages.

Table 2. Diversity of local practices around drillings management.

\begin{tabular}{|c|c|c|c|}
\hline Cas d'étude & Objet de la transaction & Acteurs impliqués & Organisation de la transaction \\
\hline $\begin{array}{l}\text { Forage privé - agriculteurs } \\
\text { vendeurs d'eau }\end{array}$ & $\begin{array}{l}\text { Vente de tours d'eau associée, } \\
\text { ou non, à la cession d'une } \\
\text { parcelle agricole }\end{array}$ & $\begin{array}{l}\text { Propriétaires de terres et de } \\
\text { forages vendant de l'eau à des } \\
\text { locataires installés sur leur terre, } \\
\text { mais aussi aux agriculteurs } \\
\text { voisins }\end{array}$ & $\begin{array}{l}\text { Contrat verbal pour une ou } \\
\text { plusieurs saisons (pour la } \\
\text { diversité des arrangements, cf. } \\
\text { Amichi et al., } 2015 \text {; Ouendeno } \\
\text { et al., 2015) }\end{array}$ \\
\hline
\end{tabular}

d'autorisations est officiellement gelé et les propriétaires des exploitations existantes dénoncent systématiquement toute installation informelle de chantiers de forage. L'engagement des propriétaires dans ce processus informel de contrôle et de dénonciation peut être interprété comme étant une première étape vers l'émergence d'une action collective autour de la gestion de la nappe dont le stock est réellement menacé. Le coût de la non-implication dans la gestion collective devient plus élevé que le coût de l'implication, ce qui favorise l'émergence d'une action collective, comme le montrent également les analyses d'Ostrom et al. (1999).

\subsection{Des règles qui incitent à la rationalité des usages et à la maximisation des prélèvements}

L'émergence du marché privé de l'eau, associé à la location $\mathrm{du}$ foncier ou non, semble être acceptée par tous les propriétaires de forages et les exploitants agricoles locataires. Il permet aux propriétaires de forages d'avoir une rétribution, considérée comme une rémunération légitime de l'investissement dans le forage et ses équipements, et aux locataires d'accéder à un facteur de production déterminant, sans coût dissuasif.

Dans les zones où le marché foncier locatif est actif et lié à un marché secondaire d'eau, les contrats de location imposant la technique du goutte-à-goutte, et fixant une dotation volumétrique d'eau préalablement déterminée, incitent à une utilisation rationnelle de l'eau d'irrigation à l'échelle de l'exploitation. Plus particulièrement, le marché secondaire de l'eau donne une valeur concrète au coût d'opportunité de l'eau d'irrigation ; tout volume d'eau pompé et non utilisé pour l'irrigation peut être valorisé sur le marché. Ainsi, les propriétaires rationalisent (au sens d'amélioration de l'efficience de l'irrigation) leur usage propre et incitent les locataires à faire de même à l'échelle de la parcelle.
Cependant, l'existence de marchés privés de l'eau et de droits d'eau non contrôlés entraîne une valorisation marchande de l'eau, au-delà des capacités d'utilisation propre de chaque propriétaire de forage. Le fonctionnement actuel du système de gouvernance de l'eau incite en effet les propriétaires de forage à maximiser les prélèvements d'eau sur le forage, pour l'usage individuel ou pour la vente. Aucune mesure formelle directe (paiement d'une redevance indexée au volume pompé) ou indirecte (écart important entre la tarification des tranches de consommation d'énergie électrique) n'incite les propriétaires des forages à réduire les volumes pompés. En Asie, Shah (2009) proposait de limiter les prélèvements par des incitations indirectes, notamment en augmentant les coûts de l'énergie. En Algérie, pays pétrolier, de telles mesures seraient compliquées à mettre en œuvre, l'électricité étant largement subventionnée, notamment au sud du pays. D'autres mesures de tarification doivent être explorées. L'existence de règles informelles visant à rationaliser les usages et associées au rôle effectif de l'État tend à montrer que le paiement d'une redevance à l'État pour le pompage de l'eau souterraine par les agriculteurs pourrait également être une mesure efficace, acceptée par les agriculteurs. Elle permettrait d'inciter à limiter les prélèvements sur la nappe, sans être un frein au développement agricole. Si la redevance, prévue par la loi de 2005, est étudiée, justifiée et légitimée, elle peut être un instrument de contrôle et de gestion efficace des prélèvements de l'eau souterraine. Sa justification et sa légitimation auprès des usagers de l'eau ne peuvent se faire qu'à deux conditions :

- leur implication dans la conception et la mise en œuvre du dispositif de prélèvement et d'affectation de la redevance ;

- la redevance doit servir, au moins en partie, à payer des actions d'économie d'eau (améliorer les infrastructures et équipements d'irrigation) et ou de développement agricole au bénéfice des usagers qui paient. 


\section{Conclusion}

Dans cet article, nous montrons que la dynamique de l'agriculture dans la région est principalement permise par un complexe jeu d'acteurs, au niveau bilatéral et collectif, qui arrivent à reconfigurer et à adapter le cadre institutionnel régissant l'usage de la ressource en eau. L'écart et la complémentarité existant entre le cadre formel et les pratiques locales d'accès à l'eau, malgré leurs limites, contribuent à la dynamique agricole du Sahara algérien. À l'échelle des exploitations, l'existence de marchés de l'eau et de droits d'eau associés au foncier locatif incitent à la rationalité des usages sur les parcelles et à la maximisation des prélèvements sur le forage. L'État est « tolérant », mais non absent de cette dynamique, et pourrait reprendre en partie la main sur le contrôle de l'eau, via le contrôle des autorisations de forages et via une redevance.

La baisse de niveau des nappes souterraines exploitées au Sahara (Côte, 2011) reste toutefois une menace sérieuse pour la pérennité de la nouvelle agriculture saharienne. Toutes les sources artésiennes de la région d'étude, qui faisaient autrefois sa spécificité, sont aujourd'hui taries. L'irrigation se fait exclusivement à partir de forages de plus en plus profonds. Le renouvellement de ces nappes étant limité, le risque d'épuisement, à terme, est donc réel et avec lui les risques d'effondrement de toute l'agriculture, traditionnelle d'abord et nouvelle ensuite, dans cette partie du Sahara. Comme dans d'autres régions d'Algérie où les baisses du niveau des nappes mettent en péril les exploitations, l'émergence d'une action collective portée par les propriétaires de la zone est possible. Cette action collective aurait les moyens de s'appuyer sur des leviers réglementaires existants et sur des règles locales informelles pour limiter les autorisations de forages et espérer réguler les usages.

\section{Références}

Amichi F, Bouarfa S, Lejars C, Kuper M, Hartani T, Daoudi A, et al. 2015. Des serres et des hommes : des exploitations motrices de l'expansion territoriale et de l'ascension socioprofessionnelle sur un front pionnier de l'agriculture saharienne en Algérie. Cahiers Agricultures 24(1): 11-19. DOI: 10.1684/agr.2015.0736.

APC d'El Ghrous. 2012. Données statistiques des services agricoles de l'Assemblée populaire communale d'El Ghrous.

Bensaâd A. 2011. Le Sahara, une civilisation hydraulique en creux. In : Bensaâd A, ed. L'eau et ses enjeux au Sahara. Paris : Édition Karthala.

Bessaoud O. 2013. La question foncière au Maghreb : la longue marche vers la privatisation. Les Cahiers du Cread 103: 17-44.

Bouammar B. 2010. Le développement agricole dans les régions sahariennes. Étude de cas de la région de Ouargla et de Biskra. Thèse de doctorat en sciences économiques. Alger: Université de Ouargla.

Brochier-Puig J. 2004. Société locale et état face aux limites de la ressource eau (Nefzaoua, sud-ouest tunisien). In : Picouet M, Sghaier M, Genin D, Abaab A, Guillaume H, Elloumi M, eds. Environnement et sociétés rurales en mutation : approches alternatives. Paris : IRD, pp. 307-321, ISBN 2-7099-1547-2.

Côte M. 2002. Des oasis aux zones de mises en valeur : l'étonnant renouveau de l'agriculture saharienne. Méditerranée 99: 5-14. DOI: $10.3406 /$ medit.2002.3253.
Côte M. 2011. L'eau au Sahara, nouvelles potentialités et nouvelles interrogations. In : Bensaâd A, ed. L'eau et ses enjeux au Sahara. Paris : Édition Karthala.

Daoudi A, Lejars C. 2016. De l'agriculture oasienne à l'agriculture saharienne dans la région des Ziban en Algérie : acteurs du dynamisme et facteurs d'incertitude. New Medit 15: 45-52.

Daoudi A, Colin JP, Derderi A, Ouendeno ML. 2015. Mise en valeur agricole et accès à la propriété foncière en steppe et au Sahara (Algérie). Les Cahiers du Pôle Foncier 13, 34 p.

Daoudi A, Colin JP, Derderi A, Ouendeno ML. 2017. Le marché $\mathrm{du}$ faire-valoir indirect comme vecteur de nouvelles formes d'exploitation dans la néo-agriculture saharienne (Algérie). Géographie Economie Société 18: 49-72.

DSA, 2013. Monographie agricole de la wilaya de Biskra. Direction des services agricoles (DSA) de la Wilaya de Biskra.

Easter KW, Rosegrant MW, Dinar A. 1999. Formal and informal markets for water: institutions, performance, and constraints. The World Bank Research Observer 14(I): 99-116.

Faysse N, Petit O. 2012. Convergent readings of groundwater governance? Engaging exchanges between different research perspectives. Irrigation and Drainage 61: 106-114.

Fofack R, Kuper M, Petit O. 2015. Hybridation des règles d'accès à l'eau souterraine dans le Saiss (Maroc) : entre anarchie et Léviathan? Études Rurales 196: 127-150.

Granier JC. 1980. Rente foncière en eau et régulation économique dans le Gourara algérien. Revue Tiers-Monde XXI(83): 649-663.

Hamamouche MF, Kuper M, Lejars C. 2015. Émancipation des jeunes des oasis du Sahara algérien par le déverrouillage de l'accès à la terre et à l'eau. Cahiers Agricultures 24(6): 412-419. DOI: 10.1684/agr.2015.0777.

Jenifer A, Arul C. 2012. Groundwater management: a policy perspective. World Academy of Science, Engineering and Technology 62: 303-310.

Khiari A. 2002. Une région pionnière dans le Sahara algérien : El Ghrous. Méditerranée 99: 27-30. DOI: 10.3406/medit.2002.3256.

Kuper M, Hammani A, Chohin A, Garin P, Saaf M. 2012. When groundwater takes over: linking 40 years of agricultural and groundwater dynamics in a large-scale irrigation scheme in Morocco. Irrigation and Drainage 61(S1): 45-53.

Kuper M, Faysse N, Hammani A, Hartani T, Marlet S, Hamamouche MF, et al. 2016. Liberation or anarchy? The Janus nature of groundwater use on North Africa's new irrigation frontiers. In: Jakeman T, Barreteau O, Hunt R, Rinaudo JD, Ross A, eds. Integrated Groundwater Management. Dordrecht: Springer, pp. 583-615.

Llamas M, Martinez-Santos P. 2012. Intensive groundwater use: silent revolution and potential source of social conflits. $J$ Water Resour Plann Manage 131(3): 337-341.

Marouf N. 1980. Lecture de l'espace oasien. Paris : Sindbad, La Bibliothèque arabe, coll. " Hommes et sociétés », 281 p.

Mubarak JA. 1998. Middle East and North Africa: development policy in view of a narrow agricultural natural resource base. World development 26(5): 877-895.

Ostrom E. 1990. Governing the commons: the evolution of institutions for collective action. New York: Cambridge University Press.

Ostrom E. 1992. Crafting institutions for self-governing irrigation systems. San Francisco: Institute for Contemporary Studies Press, $111 \mathrm{p}$.

Ostrom E, Burger J, Field C, Norgaard R, Policansky D. 1999. Revisiting the commons: local lessons, global challenges. Review Sustainability 284: 278-282. 
Otmane T, Kouzmine Y. 2013. Bilan spatialisé de la mise en valeur agricole au Sahara algérien, Cybergeo. European Journal of Geography [En ligne], Espace, Société, Territoire, article 632, mis en ligne le 19 février 2013, consulté le 20 février 2013. Disponible sur : http://cybergeo.revues.org/25732.:10.4000/cybergeo.25732.

Ouendeno ML, Daoudi A, Colin JP. 2015. Les trajectoires professionnelles des jeunes dans la néo-agriculture saharienne (Biskra, Algérie) revisitées par la théorie de l'agricultural ladder. Cahiers Agricultures 24: 396-403. DOI: 10.1684/agr.2015.0793.

Petit O. 2004. La surexploitation des eaux souterraines : enjeux et gouvernance. Natures sciences sociétés 2(12): 146-156.

Petit O. 2016. Paradise lost? The difficulties in defining and monitoring integrated water resoures management indicators. Current opinion in environmental sustainability 21: 58-64.
Petit O, Kuper M, López-Gunn E, Rinaudo JD, Daoudi A, Lejars C. 2017. Can agricultural groundwater economies collapse? An inquiry into the pathways of four groundwater economies under threat. Hydrogeology Journal, 16 p. DOI: 10.1007/s10040-0171567-3.

Quarouch H, Kuper M, Abdellaoui EH, Bouarfa S. 2014. Eaux souterraines, sources de dignité et ressources sociales : cas d'agriculteurs dans la plaine du Saïss au Maroc. Cahiers Agricultures 23: 158-65. DOI: 10.1684/agr.2014.0699.

Shah T. 2009. Taming the anarchy: groundwater governance in South Asia. Washington DC: Resources for the Future Press.

Siebert S, Burke J, Faures JM, Frenken K, Hoogeveen J, Doll P, et al. 2010. Groundwater use for irrigation - a global inventory. Hydrology Earth System Science 14: 1863-1880.

Citation de l'article : Daoudi A, Lejars C, Benouniche N. 2017. La gouvernance de l'eau souterraine dans le Sahara algérien : enjeux, cadre légal et pratiques locales. Cah. Agric. 26: 35004. 